\title{
Efficient materials for spin-to-orbit angular momentum conversion using bending technique
}

\author{
Vasylkiv Yu., Smaga I., Skab I. and Vlokh R. \\ Institute of Physical Optics, 23 Dragomanov Street, 79005 Lviv, Ukraine, \\ vlokh@ifo.lviv.ua
}

Received: 10.09 .2013

\begin{abstract}
We analyze the efficiency of different materials used for spin-to-orbit angular momentum conversion by applying bending to parallelepiped-shaped bars made of crystals or glasses. It is found that generation of pure screw dislocations of the light wave front with the bending technique is possible only in isotropic materials and crystals belonging to hexagonal and trigonal symmetries, with exception of crystals of the point symmetry groups $3, \overline{3}, 6, \overline{6}$ and $6 / \mathrm{m}$. We demonstrate that lexan is the most efficient material for generating optical vortex beams that bear orbital angular momentum, using the bending technique. This material reveals the highest figure of merit, $|\mathfrak{M}|=155.1 \times 10^{-12} \mathrm{~m}^{2} / \mathrm{N}$.
\end{abstract}

Keywords: optical vortex, efficiency of SAM-to-OAM conversion, bending

PACS: $42.50 . \mathrm{Tx}, 42.70 .-\mathrm{a}, 78.20 . \mathrm{hb}$

UDC: $535.5+535.012+535.55+539.384+517.951 .5$

\section{Introduction}

Optical vortices in the light beams that bear orbital angular momentum can be generated by a number of techniques. Usually they are based on computer-synthesized holograms with fork-like defects or spiral patterns of gratings [1, 2], spiral phase plates [3], optical wedges [4], q-plates [5, $6]$, etc. All of those tools for generating optical vortices, except for the q-plates, represent so-called passive techniques. In other words, the efficiency of spin-to-orbit angular momentum (SAM-toOAM) conversion cannot be operated by external influences, which represents a serious drawback. Only the efficiency of the SAM-to-OAM conversion with the q-plates, which represent liquid crystalline matrices with embedded defects in their centres, can be tuned by either temperature or electric field $[7,8]$.

Recently we have shown that optical vortices can also be generated with the aid of parametric optical effects (e.g., piezooptic or Pockels ones) induced by inhomogeneous external fields [9-12]. For example, optical polarization singularities are successfully created using torsion of crystals, their bending [9-11], or loading of transparent crystalline disks compressed along their diameters [12]. It has also been found that optical polarization singularities are imposed by structural dislocations existing in crystals or by residual stresses available in glass materials [13]. Moreover, singularities with the strength of topological defects equal to $1 / 2$ can be induced by conically shaped electric field via the Pockels effect [14].

Keeping all of those new techniques in mind, one can infer that searching for efficient materials for generation of the optical vortices on the basis of parametric optical effects represents an urgent practical problem. In our recent works $[15,16]$ we have shown that, in what refers the torsion effect, the crystals of $\mathrm{LiNbO}_{3}, \beta-\mathrm{BaB}_{2} \mathrm{O}_{4}$ and $\alpha-\mathrm{BaB}_{2} \mathrm{O}_{4}$ are the most efficient materials, 
with their figure of merit being as high as of the order of $|\mathfrak{M}| \sim 10^{-11} \mathrm{~m}^{2} / \mathrm{N}[15]$. On the other side, $\mathrm{Bi}_{12} \mathrm{TiO}_{20}$ crystals are the most efficient while inducing the orbital angular momentum by the electric field via the Pockels effect [16]. They reveal the highest value of the relevant figure of merit, $|\mathfrak{M}|=7.8 \times 10^{-11} \mathrm{~m} / \mathrm{V}$. However, the Pockels effect exists only in acentric material media, while the torsion-induced optical vortices can be generated only in the crystals belonging to trigonal or cubic symmetry groups. These points significantly restrict the number of materials used for this aim.

On the other hand, optical vortices can be generated by bending a bar made of some material even with isotropic media, e.g., glasses [11]. Thus, we believe that analysis of efficiency of the SAM-to-OAM conversion for different materials subjected to bending is very important. The present work is just devoted to this problem.

\section{Conditions for generating canonical vortices under bending of a bar with paral- lelepiped shape}

As already shown in Ref. [11], the canonical optical vortices can be generated by bending material bars under specific geometrical conditions. Let us remind in brief the principal results of the analysis [11]. Generation of a pure screw dislocation of the phase front by bending of bars can be implemented for the two limiting cases: (i) a mechanical load is distributed over all the upper surface of a sample (see Fig. 1a), and (ii) a load is distributed over some distance $d$ along the upper surface of that sample (see Fig. 1b).
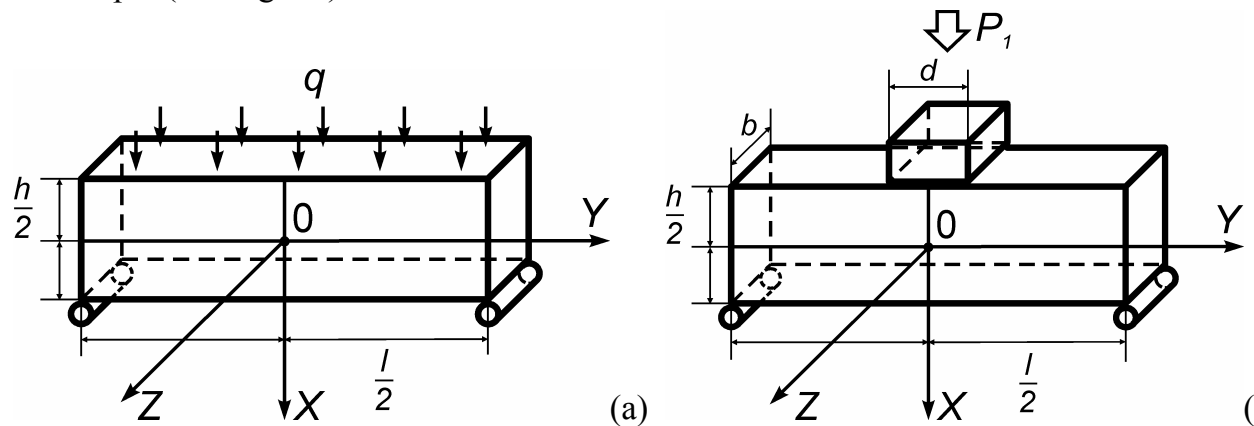

Fig.1. Schematic representation of mechanical load distributed over the entire upper sample surface (a) and over the distance $d$ at that surface (b). The parameter $q$ is defined as $q=P_{1} / l$ (see also explanations in the text).

In the both cases we have the condition $\sigma_{1}<<\sigma_{2}$ for the stress tensor components, which is satisfied at $h / l<10^{-1}$. Here $h, b, l$ and $d$ are geometrical parameters explained in Fig. 1. Let us consider the first case illustrated in Fig. 1a. When the light beam propagates along $Z$ axis in an isotropic medium (e.g., a glass), one can represent the angle of optical indicatrix rotation and the optical birefringence respectively as

$$
\tan 2 \zeta_{Z}=-\frac{2 \sigma_{6}}{\sigma_{2}}=\frac{2\left(\frac{h^{2}}{4}-X^{2}\right) Y}{\left(\frac{l^{2}}{4}-Y^{2}\right) X}=\frac{2\left(\frac{h^{2}}{4}-\rho^{2} \cos ^{2} \varphi\right)}{\left(\frac{l^{2}}{4}-\rho^{2} \sin ^{2} \varphi\right)} \tan \varphi
$$

and 


$$
\Delta n_{12}=-\frac{3 n_{o}^{3} q \pi_{66}}{b h^{3}} \sqrt{\left(\frac{l^{2}}{4}-Y^{2}\right)^{2} X^{2}+4\left(\frac{h^{2}}{4}-X^{2}\right)^{2} Y^{2}},
$$

where $n_{o}$ denotes the ordinary refractive index and $\pi_{66}=\pi_{44}=\pi_{11}-\pi_{12}$ the piezooptic coefficient. Here obvious relations $X=\rho \cos \varphi$ and $Y=\rho \sin \varphi$ between the polar and Cartesian coordinates can be used, where $\varphi$ is the polar angle and $\rho$ the module. Under the condition $\rho \rightarrow 0$, Eq. (1) may be simplified to

$$
\tan 2 \zeta_{Z}=\frac{2 h^{2}}{l^{2}} \tan \varphi
$$

which corresponds to a mixed screw-edge dislocation of the phase front. Considering that $X, Y \rightarrow 0$ at $l=h \sqrt{2}$, one can rewrite Eqs. (1) and (2) as

$$
\Delta n_{12}=-\frac{3 n_{o}^{3} P_{1} \pi_{66}}{2 \sqrt{2} b h^{3}} \sqrt{X^{2}+Y^{2}}
$$

and

$$
\tan 2 \zeta_{Z}=\tan \varphi\left(\text { or } \zeta_{Z}=\varphi / 2\right),
$$

where $P_{1}$ implies the loading force. The above relations testify that the birefringence is distributed conically. Therefore a pure screw dislocation of the phase front and a canonical vortex with a unit charge should appear. However, the condition $l=h \sqrt{2}$ contradicts the inequality $h / l<10^{-1}$ assumed above and so it cannot be realized in practice.

In the second case when the load is distributed over the distance $d(-d / 2<Y<d / 2-$ see Fig. 1b), the optical birefringence and the optical indicatrix rotation angle read as

$$
\tan 2 \zeta_{Z}=\frac{2\left(h^{2} / 4-X^{2}\right) Y}{\left(d(2 l-d) / 4-Y^{2}\right) X}=\frac{2\left(h^{2} / 4-\rho^{2} \cos ^{2} \varphi\right)}{\left(d(2 l-d) / 4-\rho^{2} \sin ^{2} \varphi\right)} \tan \varphi
$$

and

$$
\Delta n_{12}=-\frac{3 n_{o}^{3} P_{1}}{b h^{3} d} \pi_{66} \sqrt{\left(\frac{d(2 l-d)}{4}-Y^{2}\right)^{2} X^{2}+4\left(\frac{h^{2}}{4}-X^{2}\right)^{2} Y^{2}} .
$$

At small enough $\rho$ values, formula (6) reduces to

$$
\tan 2 \zeta_{Z}=\frac{2 h^{2}}{d(2 l-d)} \tan \varphi .
$$

This dependence of $\zeta_{Z}$ on the tracing angle $\varphi$ corresponds to a so-called elliptical vortex appearing due to a mixed screw-edge dislocation of the wave front. Under the condition

$$
2 h^{2} / d(2 l-d)=1\left(\text { or } d=l-\sqrt{l^{2}-2 h^{2}}\right)
$$

Eq. (8) yields in

$$
\tan 2 \zeta_{Z}=\tan \varphi\left(\text { or } \zeta_{Z}=\varphi / 2\right)
$$

i.e. we deal with a pure screw dislocation of the phase front leading to canonical vortex that has a unit charge. Under the same conditions, the spatial distribution of the birefringence is given by a conical shape: 


$$
\Delta n_{12}=-\frac{3 n_{o}^{3} P_{1} l}{2 b h^{3}} \pi_{66} \sqrt{X^{2}+Y^{2}} .
$$

Since the geometrical relation mentioned above can be simply reached, further on we will consider only the second case.

\section{Relation for the efficiency of SAM-to-OAM conversion}

Following our recent results $[15,16]$, one can represent the efficiency of the SAM-to-OAM conversion as

$$
\eta=\frac{I_{l}^{\text {out }}}{I_{r}^{\text {inc }}}
$$

where $I_{r}^{\text {inc }}$ and $I_{l}^{\text {out }}$ are the intensities of respectively the right-handed incident light wave and the left-handed outgoing wave that bears OAM.

Let us write out the relation describing the $X Y$ cross section of the Fresnel ellipsoid perturbed by the stress tensor components $\sigma_{2}$ and $\sigma_{6}$ for the cubic crystals, under the condition that the optical beam propagates along the $Z$ axis:

$$
\left(B_{1}+\pi_{12} \sigma_{2}\right) X^{2}+\left(B_{1}+\pi_{11} \sigma_{2}\right) Y^{2}+2 \pi_{44} \sigma_{6} X Y=1,
$$

where the stress tensor components are given by the relations [11]

$$
\sigma_{2}=\frac{6 P_{1}}{b h^{3}} \frac{d(2 l-d)-4 Y^{2}}{4 d} X, \sigma_{6}=-\frac{6 P_{1} Y}{b h^{3} d}\left(\frac{h^{2}}{4}-X^{2}\right) .
$$

Then the angle of optical indicatrix rotation reduces to

$$
\tan 2 \zeta_{Z}=\frac{2 \pi_{44}}{\left(\pi_{12}-\pi_{11}\right)} \frac{\sigma_{6}}{\sigma_{2}} .
$$

Since the relation $\pi_{66}=\pi_{44} \neq \pi_{11}-\pi_{12}$ holds true for the cubic crystals, the elliptical vortex would appear even in the case when Eq. (8) is satisfied. Thus, one has to choose optically uniaxial crystalline materials of such point symmetry groups for which the condition $\pi_{66}=\pi_{11}-\pi_{12}$ fulfils. These groups are as follows: $3, \overline{3}, 32,3 \mathrm{~m}, \overline{3} \mathrm{~m}, 6, \overline{6}, 6 / \mathrm{m}, \overline{6} \mathrm{~m} 2,622,6 \mathrm{~mm}$, and 6/mmm [17]. However, the crystals belonging to the symmetry groups $3, \overline{3}, 6, \overline{6}$ and $6 / \mathrm{m}$ manifest a very complicated structure of the piezooptic tensor. The consequence is a complicated relation for the optical indicatrix,

$$
\left(B_{1}+\pi_{12} \sigma_{2}+2 \pi_{62} \sigma_{6}\right) X^{2}+\left(B_{1}+\pi_{11} \sigma_{2}-2 \pi_{62} \sigma_{6}\right) Y^{2}++2\left(\pi_{62} \sigma_{2}+\pi_{66} \sigma_{6}\right) X Y=1,
$$

which yields in the following optical indicatrix rotation:

$$
\tan 2 \zeta_{Z}=\frac{2\left(\pi_{62} \sigma_{2}+\pi_{66} \sigma_{6}\right)}{4 \pi_{62} \sigma_{6}-\pi_{66} \sigma_{2}}
$$

One can see that Eq. (17) implies generation of the screw-edge dislocation of the wave front.

Summarizing all the considerations mentioned above, we conclude that the pure screw dislocation can be generated by the bending technique only in the isotropic materials and the crystals that belong to the hexagonal and trigonal symmetries, with exception of crystals belonging to the point groups $3, \overline{3}, 6, \overline{6}$ and $6 / \mathrm{m}$. In these cases only the coefficient $\pi_{66}=\pi_{11}-\pi_{12}$ is involved into piezooptic interaction.

Following the results $[15,16]$, we will obtain the relation needed for calculating the SAM-to- 
OAM conversion efficiency. For this aim we divide the optical beam into $k \times l=N$ elementary rays, while the intensity of the incoming wave is taken to be unit ( $I_{r}^{i n c}=1$ ). Then Eq. (12) may be represented as

$$
\eta=\frac{\sum_{k=1}^{\sqrt{N}} \sum_{l=1}^{\sqrt{N}}\left(I_{l}^{\text {out }}\right)_{k l}}{N} .
$$

Here the sample is divided into $k \times l$ homogeneous elementary cells in the $X Y$ plane and, in practice, we put $k=30$ and $l=30$. The size of each square homogeneous elementary cell in the $X Y$ plane is taken to be equal to $0.032 \times 0.032 \mathrm{~mm}^{2}$. Since the beam has a circular cross section rather than a square one, the number of square-shaped elementary cells packed in such a cross section will be somewhat smaller than $N=900$. Suppose now that the light beam propagates through a system of mutually orthogonally oriented circular polarizers and a crystalline sample subjected to bending and placed in between the polarizers. Then one gets the relation

$$
\left(I_{l}^{\text {out }}\right)_{k l}=\left(I_{r}^{\text {inc }}\right)_{k l} \sin ^{2} \Delta \Gamma / 2 \text {. }
$$

With considering Eq.(7), the phase difference is determined as

$$
\begin{aligned}
\Delta \Gamma & =\frac{2 \pi b}{\lambda} \Delta n_{12}=\frac{6 \pi}{\lambda} \frac{n_{o}^{3} P_{1}}{h^{3} d} \pi_{66} \sqrt{\left(\frac{d(2 l-d)}{4}-Y^{2}\right)^{2} X^{2}+4\left(\frac{h^{2}}{4}-X^{2}\right)^{2} Y^{2}} \\
& =\frac{6 \pi}{\lambda h^{3} d} \mathfrak{M} P_{1} \sqrt{\left(\frac{d(2 l-d)}{4}-Y^{2}\right)^{2} X^{2}+4\left(\frac{h^{2}}{4}-X^{2}\right)^{2} Y^{2}},
\end{aligned}
$$

where $\mathfrak{M}=n_{o}^{3} \pi_{66}$ is a parameter that would define the efficiency of the SAM-to-OAM conversion, i.e. a figure of merit.

Using Eq. (18), one arrives at the following expression for the efficiency of the SAM-toOAM conversion:

$$
\eta=\left.\frac{\sum_{X=-R}^{R} \sum_{Y=-R}^{R} \sin ^{2}\left(\frac{6 \pi}{\lambda h^{3} d} \mathfrak{M} P_{1} \sqrt{\left(\frac{d(2 l-d)}{4}-Y^{2}\right)^{2} X^{2}+4\left(\frac{h^{2}}{4}-X^{2}\right)^{2} Y^{2}}\right)}{N}\right|_{X^{2}+Y^{2} \leq R^{2}},
$$

where the practical step taken for the $X$ and $Y$ coordinate changes is equal to $0.032 \mathrm{~mm}$, and $R$ is the beam radius. Hence, the efficiency of the SAM-to-OAM conversion represents a quantity integrated over the cross section of the wide light beam. As a consequence, the problem of searching for materials efficient for the SAM-to-OAM conversion under the action of bending has been reduced to searching for crystalline materials of the trigonal and hexagonal symmetries or glass materials, which reveal the highest figures of merit $\mathfrak{M}$. In the presence of natural optical activity effect, one should additionally take into account the phase difference caused by the optical rotation. It is the same for all the elementary cells: $\Delta \Gamma_{o a}=2 \pi b g_{i} / \lambda n_{o}$, where $g_{i}$ is the gyration tensor component along the direction of light propagation. 


\section{Estimations of efficiency of the SAM-to-OAM conversion for different materials}

Table 1 presents the piezooptic parameters important for the SAM-to-OAM conversion in some crystals and glasses whose optical characteristics are well known. As seen from Table 1, the highest figure of merit among the crystalline materials is typical for $\operatorname{ZnSe}\left(|\mathfrak{M}|=29.9 \times 10^{-12} \mathrm{~m}^{2} / \mathrm{N}\right)$. Among the inorganic glasses, a fluoro-crown glass FK7 reveals the highest figure of merit. However, the polymer compounds, in particular lexan, are characterized by still better characteristics. The figure of merit for the latter substance is two orders of magnitude higher than that found for the other materials.

Table 1. Parameters defining the efficiency of the SAM-to-OAM conversion under bending of material bars (at the light wavelength $\lambda=632.8 \mathrm{~nm}$ ).

\begin{tabular}{|c|c|c|c|c|c|}
\hline $\begin{array}{l}\text { Point sym- } \\
\text { metry group }\end{array}$ & Crystal & $\begin{array}{l}\text { Piezooptic coefficient } \pi_{66}, \\
10^{-12} \mathrm{~m}^{2} / \mathrm{N} \text {; gyration tensor com- } \\
\text { ponent, and references }\end{array}$ & $\begin{array}{l}\text { Refractive } \\
\text { index } n_{o} \text {, } \\
\text { and refer- } \\
\text { ence }\end{array}$ & $\begin{array}{l}\text { Figure of } \\
\text { merit } \\
|\mathfrak{M}|, \\
10^{-12} \mathrm{~m}^{2} / \mathrm{N}\end{array}$ & $\begin{array}{l}P_{1}, \mathrm{~N} \\
\text { corre- } \\
\text { spondding } \\
\text { to } \eta_{\max }\end{array}$ \\
\hline $6 \mathrm{~mm}$ & $\mathrm{ZnSe}$ & $\pi_{66}=\pi_{11}-\pi_{12}=-1.7[18]$ & $\sim 2.6[18]$ & 29.9 & 24 \\
\hline$\overline{3} \mathrm{~m}$ & $\alpha-\mathrm{Al}_{2} \mathrm{O}_{3}$ & $\pi_{66}=\pi_{11}-\pi_{12}=0.45[18]$ & $1.76[18]$ & 2.45 & 312 \\
\hline $3 m$ & $\mathrm{LiNbO}_{3}$ & $\pi_{66}=\pi_{11}-\pi_{12}=0.62[19]$ & $2.286[18]$ & 7.41 & 102 \\
\hline $3 \mathrm{~m}$ & $\mathrm{LiTaO}_{3}$ & $\pi_{66}=\pi_{11}-\pi_{12}=-0.96[20]$ & $2.175[18]$ & 9.88 & 78 \\
\hline 32 & $\mathrm{La}_{3} \mathrm{Ga}_{5} \mathrm{SiO}_{14}$ & $\begin{array}{l}\pi_{66}=\pi_{11}-\pi_{12}=-0.29[21] ; \\
g_{3}=2.2 \times 10^{-5}[22]\end{array}$ & $\begin{array}{l}1.8993 \\
{[21]}\end{array}$ & 1.99 & 396 \\
\hline 32 & $\mathrm{SiO}_{2}$ & $\begin{array}{l}\pi_{66}=\pi_{11}-\pi_{12}=-1.34 \\
g_{3}=12.9 \times 10^{-5}[18]\end{array}$ & $1.542[18]$ & 4.91 & 390 \\
\hline$\infty / \infty / \mathrm{mmm}$ & $\begin{array}{l}\text { Polymethyl } \\
\text { methacrylate } \\
\text { (PMMA) }\end{array}$ & $\begin{array}{l}\pi_{66}=\pi_{44}=\pi_{11}-\pi_{12}=2.64 \\
{[23]}\end{array}$ & $1.49[23]$ & 8.73 & 90 \\
\hline$\infty / \infty / \mathrm{mmm}$ & Plexiglas 55 & $\pi_{66}=\pi_{44}=\pi_{11}-\pi_{12}=1.2[24]$ & $\begin{array}{l}1.4934 \\
{[24]}\end{array}$ & 4.0 & 192 \\
\hline$\infty / \infty / \mathrm{mmm}$ & Lexan & $\begin{array}{l}\pi_{66}=\pi_{44}=\pi_{11}-\pi_{12}=-39.2 \\
{[24]}\end{array}$ & $\begin{array}{l}1.5816 \\
{[24]}\end{array}$ & 155.1 & 5 \\
\hline$\infty / \infty / \mathrm{mmm}$ & $\begin{array}{l}\text { Borosilicate } \\
\text { glass } \\
\text { BK7 }\end{array}$ & $\begin{array}{l}\pi_{66}=\pi_{44}=\pi_{11}-\pi_{12}=-1.59 \\
{[25]}\end{array}$ & $\begin{array}{l}1.5151 \\
{[26]}\end{array}$ & 5.53 & 138 \\
\hline$\infty / \infty / \mathrm{mmm}$ & $\begin{array}{l}\text { Fluoro- } \\
\text { crown glass } \\
\text { FK7 }\end{array}$ & $\begin{array}{l}\pi_{66}=\pi_{44}=\pi_{11}-\pi_{12}=2.49 \\
{[26]}\end{array}$ & $1.463[26]$ & 7.8 & 96 \\
\hline
\end{tabular}

The calculated dependences of the efficiency of SAM-to-OAM conversion on the loading force for different materials are presented in Fig. 2. In our calculations, the following geometric parameters have been used: $l=20.3 \mathrm{~mm}, h=3.2 \mathrm{~mm}, b=5.65 \mathrm{~mm}$, and $d=0.52 \mathrm{~mm}$. As seen from Fig. 2, the natural optical activity decreases the efficiency of the SAM-to-OAM conversion (see also [27]). This is why the crystals that belong to the symmetry group 32 will hardly be efficient in practical applications. 

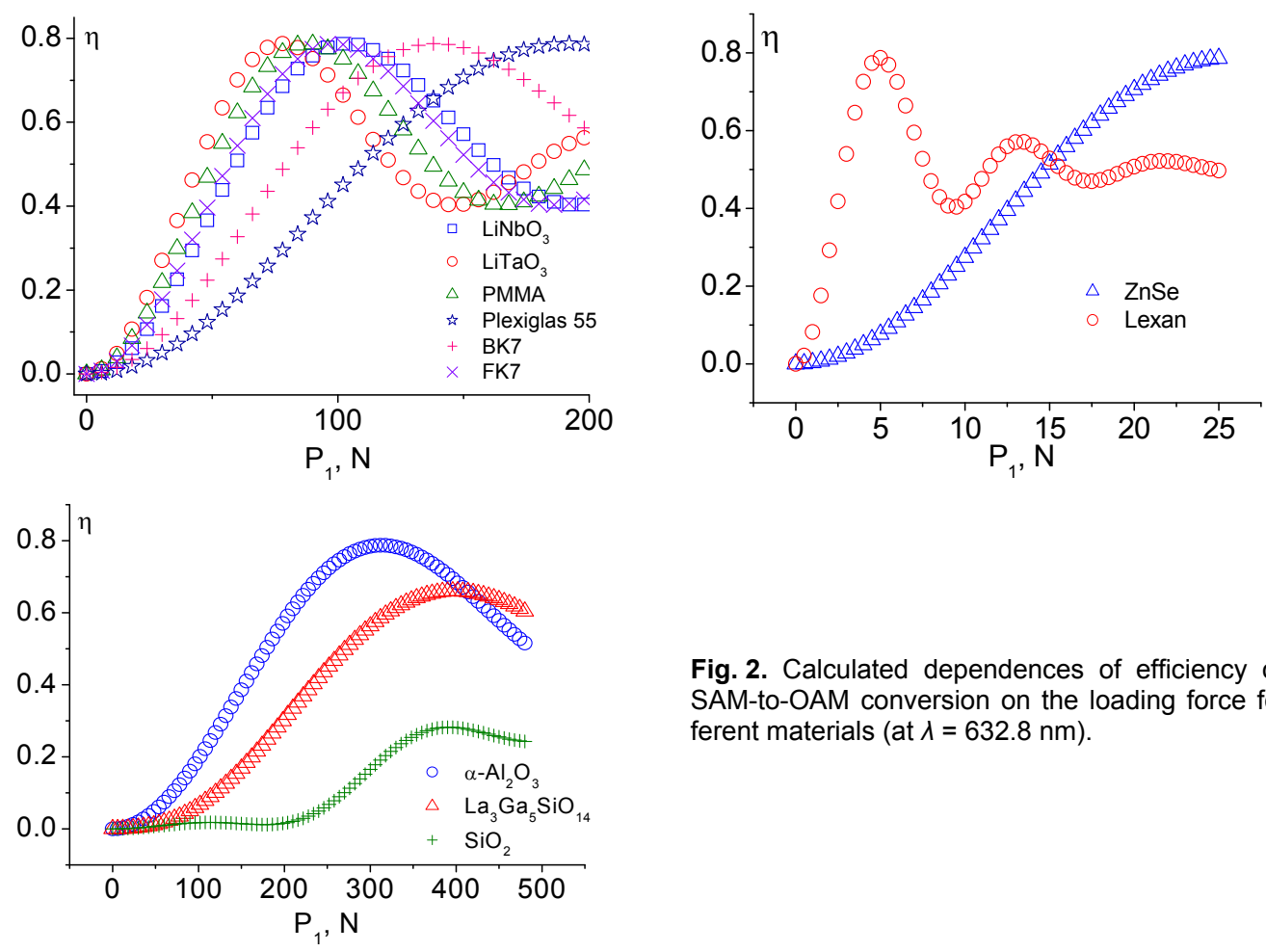

Fig. 2. Calculated dependences of efficiency of the SAM-to-OAM conversion on the loading force for different materials (at $\lambda=632.8 \mathrm{~nm}$ ).

\section{Experimental results}

To verify experimentally the appearance of polarization singularities under bending of bars, we have used a BK7 glass with the dimensions mentioned above. The polarimetric setup and the working method used for measuring the angle of optical indicatrix rotation and the phase difference have been described elsewhere [9].

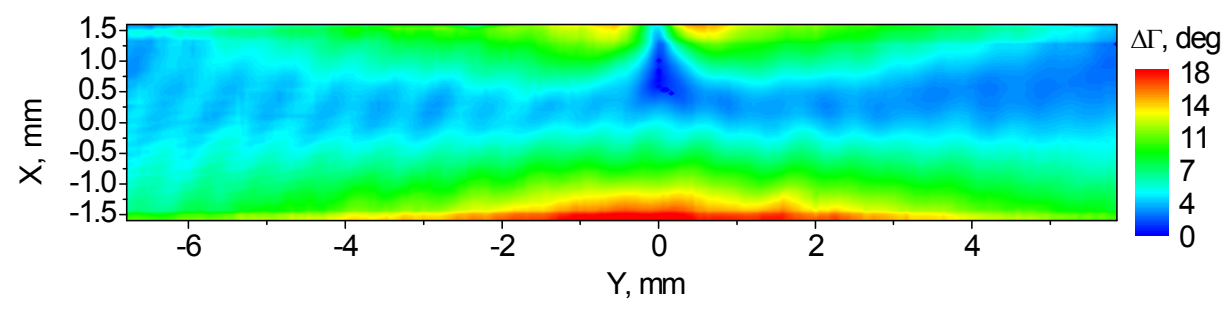

(a)

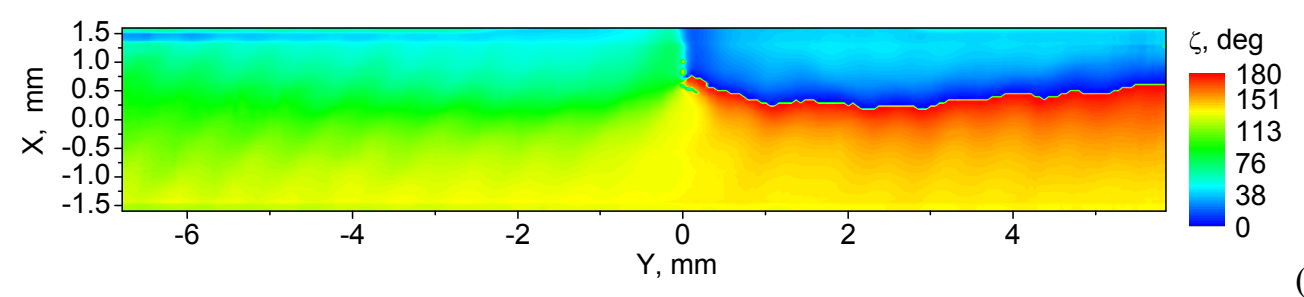

(b)

Fig. 3. Spatial distributions of the phase difference (a) and the angle of optical indicatrix rotation (b) obtained experimentally under bending of a bar made of BK7 glass (the loading force $P_{1}=5.1 \mathrm{~N}$ and $\lambda=632.8 \mathrm{~nm}$ ).

As seen from Fig. 3, the phase difference acquires a zero value approximately in the centre of the $X Y$ cross section of our sample. The angle of optical indicatrix rotation at the same point reaches a singular (i.e., indefinite) value. If the tracing angle $\varphi$ changes by $360 \mathrm{deg}$, the change in 
the angle of optical indicatrix rotation is equal to $180 \mathrm{deg}$. This corresponds to the strength $1 / 2$ of the topological defect, i.e. to generation of the singly charged optical vortex in case if the sample is placed in between the orthogonal circular polarizers. However, the linear dependence $\zeta(\varphi)$ is typical only for the distances less than $0.2 \mathrm{~mm}$ (see Fig. 4). In other words, while generating the canonical vortex, the beam radius should be smaller than $\sim 0.2 \mathrm{~mm}$. Fig. 5 presents the spatial distribution of the intensity of outgoing optical beam bearing the optical vortex, which has been simulated for the particular case of the bended BK7 glass.

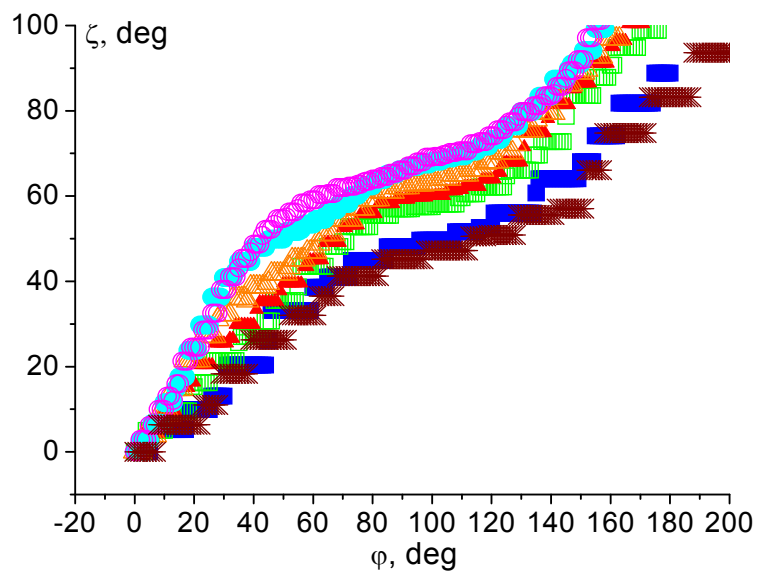

Fig. 4. Experimental dependences of angle of the optical indicatrix rotation on the tracing angle for different $\rho$ values: open circles $-\rho=1.05$, full circles -0.79 , open triangles -0.63 , full triangles -0.52 , open squares -0.42 , full squares -0.26 , and stars $-0.20 \mathrm{~mm}$.

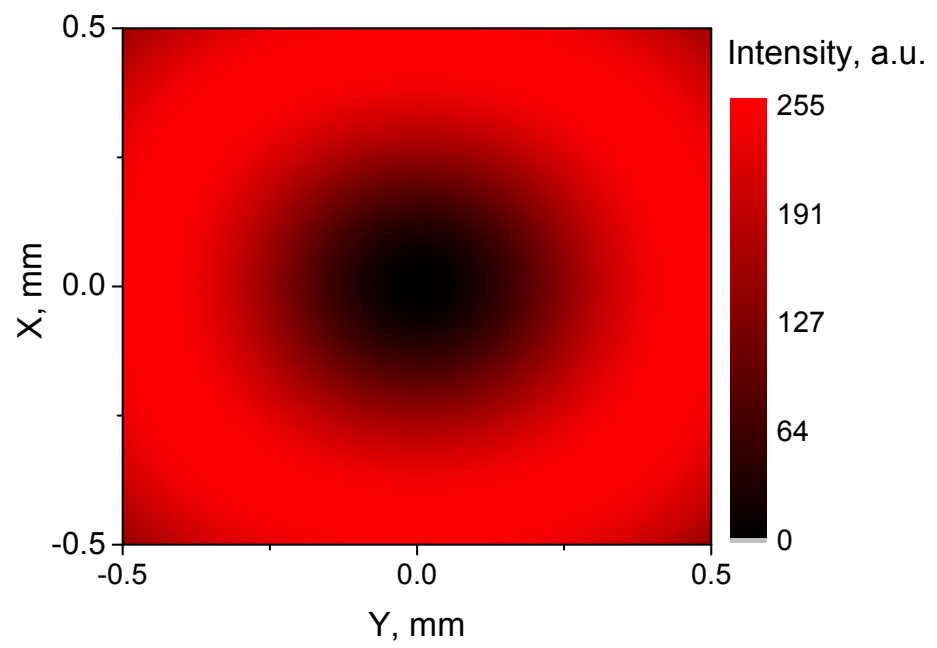

Fig. 5. Simulated spatial distribution of intensity in the outgoing optical beam bearing the optical vortex for the case of bending of the BK7 glass under the loading force $P_{1}=138 \mathrm{~N}$.

\section{Conclusions}

In the present work we have analyzed different materials utilized for optical vortex generation, using a known technique of bending of parallelepiped-shaped material bars. It has been shown that generation of the pure screw dislocation by the bending method is possible only with the isotropic materials and the crystals belonging to the hexagonal and trigonal systems, except for the crystals 
of the symmetry groups $3, \overline{3}, 6, \overline{6}$, and $6 / \mathrm{m}$. The analytical relation for the figure of merit describing the SAM-to-OAM conversion has been derived. We have demonstrated that the most efficient materials for generating optical vortex beams that bear the OAM are as follows: (i) the $\mathrm{ZnSe}$ crystals among crystalline materials (the figure of merit $|\mathfrak{M}|=29.9 \times 10^{-12} \mathrm{~m}^{2} / \mathrm{N}$ ); (ii) the fluorocrown glass FK7 among inorganic glasses $\left(|\mathfrak{M}|=7.8 \times 10^{-12} \mathrm{~m}^{2} / \mathrm{N}\right)$; and (iii) the lexan among polymer isotropic materials, with the highest figure of merit equal to $|\mathfrak{M}|=155.1 \times 10^{-12} \mathrm{~m}^{2} / \mathrm{N}$. The appearance of the optical polarization singularity under bending of the bar made of the BK7 glass has been proved experimentally.

\section{References}

1. Heckenberg N R, McDuff R, Smith C P and White A G, 1992. Generation of optical phase singularities by computer-generated holograms. Opt. Lett. 17: 221-223.

2. Soskin M S, Polyanskii P V and Arkhelyuk O O, 2004. Computer-synthesized hologrambased rainbow optical vortices. New J. Phys. 6: 196.

3. Chen Jun, Kuang Deng-Feng, Gui Min and Fang Zhi-Liang, 2009. Generation of optical vortex using a spiral phase plate fabricated in quartz by direct laser writing and inductively coupled plasma etching. Chinese Phys. Lett. 26: 014202.

4. Izdebskaya Ya, Shvedov V and Volyar A, 2005. Generation of higher-order optical vortices by a dielectric wedge. Opt. Lett. 30: 2472-2474.

5. Marrucci L, 2008. Generation of helical modes of light by spin-to-orbital angular momentum conversion in inhomogeneous liquid crystals. Mol. Cryst. Liq. Cryst. 488: 148-162.

6. Slussarenko S, Murauski A, Du T, Chigrinov V, Marrucci L and Santamato E, 2011. Tunable liquid crystal q-plates with arbitrary topological charge. Opt. Express. 19: 4085-4090.

7. Piccirillo B, D'Ambrosio V, Slussarenko S, Marrucci L and Santamato E, 2010. Photon spinto-orbital angular momentum conversion via an electrically tunable q-plate. Appl. Phys. Lett. 97: 241104.

8. Karimi E, Piccirillo B, Nagali E, Marrucci L and Santamato E, 2009. Efficient generation and sorting of orbital angular momentum eigenmodes of light by thermally tuned q-plates Appl. Phys. Lett. 94: 231124.

9. Skab I, Vasylkiv Yu, Savaryn V and Vlokh R. 2011. Optical anisotropy induced by torsion stresses in $\mathrm{LiNbO}_{3}$ crystals: appearance of an optical vortex. J. Opt. Soc. Amer. A. 28: 633640.

10. Skab I, Vasylkiv Yu, Zapeka B, Savaryn V and Vlokh R, 2011. Appearance of singularities of optical fields under torsion of crystals containing threefold symmetry axes. J. Opt. Soc. Amer. A. 28: 1331-1340.

11. Skab I, Vasylkiv Y and Vlokh R, 2012. Induction of optical vortex in the crystals subjected to bending stresses. Appl. Opt. 51: 5797-5805.

12. Skab I, Vasylkiv Y, Krupych O, Savaryn V and Vlokh R, 2012. Generation of doubly charged vortex beam by concentrated loading of glass disks along their diameter. Appl. Opt. 51: 16311637.

13. Krupych O, Vasylkiv Yu, Kvasnyuk O, Skab I and Vlokh R, 2012. Appearance of optical singularities at the light propagation through glasses with residual stresses. Ukr. J. Phys. Opt. 13: $170-176$.

14. Skab I, Vasylkiv Yu, Smaga I and Vlokh R, 2011. Spin-to-orbital momentum conversion via 
electro-optic Pockels effect in crystals. Phys. Rev. A. 84: 043815.

15. Vasylkiv Yu, Skab I and Vlokh R, 2013. Efficiency of spin-to-orbit conversion in crystals subjected to torsion stresses. Ukr. J. Phys. Opt. 14: 50-56.

16. Vasylkiv Yu, Skab I and Vlokh R, 2013. Efficiency of electrooptic spin-to-orbital angular momentum conversion in crystals. Opt. Mat. 35: 2496-2501.

17. Sirotin Yu I and Shaskolskaya M P, Fundamentals of crystal physics. Moscow: Nauka (1979).

18. Shaskolskaya M P, Acoustic crystals. Moscow: Nauka (1982).

19. Krupych O, Savaryn V, Krupych A, Klymiv I and Vlokh R, 2013. Determination of piezooptic coefficients of crystals by means of four-point bending. Appl. Opt. 52: 4054-4061.

20. Vedam K, Landolt-Börnstein - Group III Condensed matter, numerical data and functional relationships in science and technology, Ed. by D. F. Nelson, Volume 30A, Piezooptic and electrooptic constants.

21. Mytsyk B G, Demyanyshyn N M, Andrushchak A S, Kost' Ya P, Parasyuk O V and Kityk A V, 2010. Piezooptical coefficients of $\mathrm{La}_{3} \mathrm{Ga}_{5} \mathrm{SiO}_{14}$ and $\mathrm{CaWO}_{4}$ crystals: A combined optical interferometry and polarization-optical study. Opt. Mat. 33: 26-30.

22. Baturina O A, Grechushnikov B N, Kaminskiy A A, Konstantinova A F, Markosyan A A, Mill B V and Khodzhabagyan G G, 1987. Crystal-optical study of the compounds with the structure of tetragonal Ca-gallogermanate $\left(\mathrm{Ca}_{3} \mathrm{Ga}_{2} \mathrm{Ge}_{4} \mathrm{O}_{14}\right)$. Kristallografiya. 32: 406-412.

23. Meseguer F and Sanchez C, 1980. Piezobirefringence of PMMA: Optical and mechanical relaxations and influence of temperature. J. Mat. Sci. 15: 53-60.

24. Waxler R M, Horowitz D and Feldman A, 1979. Optical and physical parameters of plexiglas 55 and lexan. Appl. Opt. 18: 101-104.

25. Krupych O, Savaryn V, Skab I and Vlokh R, 2011. Interferometric measurements of piezooptic coefficients by means of four-point bending method. Ukr. J. Phys. Opt. 12: 150-159.

26. Weber M J, Handbook of optical materials. Boca Raton, London, New York, Washington: CRC Press (2003).

27. Vasylkiv Yu, Krupych O, Skab I and Vlokh R, 2011. On the spin-to-orbit momentum conversion operated by electric field in optically active $\mathrm{Bi}_{12} \mathrm{GeO}_{20}$ crystals. Ukr. J. Phys. Opt. 12: $171-179$.

Vasylkiv Yu., Smaga I., Skab I. and Vlokh R. 2013. Efficient materials for spin-to-orbit angular momentum conversion using bending technique. Ukr.J.Phys.Opt. 14: 200 - 209.

\begin{abstract}
Анотація. У роботі проаналізовано ефективність різних матеріалів, які можна використовувати для спін-орбітального перетворення оптичного кутового моменту за допомогою згину балок у формі паралелепіпеда. Виявлено, що генерачія чистої гвинтової дислокаиії хвильового фронту за методом згину можлива лише в ізотропних матеріалах $i$ кристалах, що належать до гексагональних $і$ тригональних сингоній, за винятком кристалів симетрійних груп $3, \overline{3}, 6, \overline{6}$ і 6/m. Показано, що лексан-ие найефективніший матеріал для генерації оптичних вихорів, які переносять орбітальний кутовий момент, із застосуванням методу згину. Його коефіџієнт якості найвищий $i$ дорівнює $|\mathfrak{M}|=155,1 \times 10^{-12} \mathrm{M}^{2} / \mathrm{H}$.
\end{abstract}

